\title{
Old and new cluster designs in emergency field surveys: in search of a one-fits-all solution Oleg O Bilukha
}

\author{
Address: International Emergency and Refugee Health Branch, Division of Emergency and Environmental Health Services, National Center for \\ Environmental Health, Centers for Disease Control and Prevention, 4770 Buford Hwy, Mailstop F-60, Atlanta, Georgia, 30341, USA \\ Email: Oleg O Bilukha - obilukha1@cdc.gov \\ Published: 8 July 2008 \\ Emerging Themes in Epidemiology 2008, 5:7 doi:10.1 186/1742-7622-5-7 \\ This article is available from: http://www.ete-online.com/content/5/I/7 \\ (c) 2008 Bilukha; licensee BioMed Central Ltd. \\ This is an Open Access article distributed under the terms of the Creative Commons Attribution License (http://creativecommons.org/licenses/by/2.0), \\ which permits unrestricted use, distribution, and reproduction in any medium, provided the original work is properly cited. \\ Received: 28 January 2008 \\ Accepted: 8 July 2008
}

\begin{abstract}
Introduction: Cluster surveys are frequently used to measure key nutrition and health indicators in humanitarian emergencies. The survey design of 30 clusters of 7 children $(30 \times 7)$ was initially proposed by the World Health Organization for measuring vaccination coverage, and later a design of 30 clusters of 30 children $(30 \times 30)$ was introduced to measure acute malnutrition in emergency settings. Recently, designs of 33 clusters of 6 children $(33 \times 6)$ and 67 clusters of 3 children $(67 \times$ 3) have been proposed as alternatives that enable measurement of several key indicators with sufficient precision, while offering substantial savings in time. This paper explores expected effects of using $67 \times 3,33 \times 6$, or $30 \times 7$ designs instead of a "standard" $30 \times 30$ design on precision and accuracy of estimates, and on time required to complete the survey.
\end{abstract}

Analysis: The $67 \times 3,33 \times 6$, and $30 \times 7$ designs are expected to be more statistically efficient for measuring outcomes having high design effects (e.g., vaccination coverage, vitamin A distribution coverage, or access to safe water sources), and less efficient for measuring outcomes with more within-cluster variability, such as global acute malnutrition or anemia. Because of small sample sizes, these designs may not provide sufficient levels of precision to measure crude mortality rates. Given the small number ( 3 to 7 ) of survey subjects per cluster, it may be hard to select representative samples of subjects within clusters.

The smaller sample size in these designs will likely result in substantial time savings. The magnitude of the savings will depend on several factors, including the average travel time between clusters. The $67 \times 3$ design will provide the least time savings. The $33 \times 6$ and $30 \times 7$ designs perform similarly to each other, both in terms of statistical efficiency and in terms of time required to complete the survey.

Conclusion: Cluster designs discussed in this paper may offer substantial time and cost savings compared to the traditional $30 \times 30$ design, and may provide acceptable levels of precision when measuring outcomes that have high intracluster homogeneity. Further investigation is required to determine whether these designs can consistently provide accurate point estimates for key outcomes of interest. Organizations conducting cluster surveys in emergency settings need to build their technical capacity in survey design to be able to calculate context-specific sample sizes individually for each planned survey. 


\section{Introduction}

Over the past three decades, field surveys using a cluster sample design have become a standard, popular, and widely used method of measuring key nutrition and health indicators in humanitarian emergencies [1-4]. This method was first widely popularized by the Expanded Program on Immunization (EPI) of the World Health Organization, which since 1978 has promoted using a 30 cluster design, with 7 children per cluster (popularly known as "30 $\times 7$ ") for measuring immunization coverage $[5,6]$. The field applications of this method expanded rapidly to include measuring such key emergency indicators as acute malnutrition, crude and under-5 mortality, cumulative incidence of diarrhea and respiratory disease, access to safe water, prevalence of physical signs of micronutrient deficiencies, and several others [7-10]. The challenges of measuring these indicators with sufficient precision triggered modifications to the initially proposed standard $30 \times 7$ design and led to the introduction of a recommendation for use of a 30 clusters of 30 children $(30 \times 30)$ design for measuring acute malnutrition in cluster surveys $[11,12]$. Recently published field survey manuals $[13,14]$ advocate calculating sample sizes and determining the number of clusters individually for each planned survey. This methodology would allow for achieving the required level of precision while keeping the sample size and the number of clusters to a necessary minimum, thus providing for rational and efficient use of scarce time and resources in emergency settings. Such calculations take into account not only statistical variables, such as the expected prevalence of the indicators being measured and the expected level of clustering of these indicators, but also logistics and the time required to survey households or individuals and to move from cluster to cluster. These calculations require substantial specialized expertise, which often is not available to organizations conducting surveys in emergencies [15-18]. The search, therefore, continues for the "standard" designs that would enable measurement of most of the key emergency indicators with acceptable precision while requiring less time and fewer resources than a $30 \times 30$ design. Most recently, $33 \times 6$ and $67 \times 3$ cluster survey designs have been proposed as alternatives that allow for measuring several indicators with sufficient precision, while offering substantial savings in cost, time, and manpower $[19,20]$.

In this paper, we will explore the expected statistical effects on precision of using the newly proposed $33 \times 6$ and $67 \times 3$ designs as well as the "old" $30 \times 7$ EPI design, as compared with the conventional $30 \times 30$ design. We will then discuss the situations and outcomes for which these designs may be most statistically efficient (i.e., may result in the least loss of precision compared to a $30 \times 30$ design); offer some thoughts on the potential of these designs to introduce bias into the prevalence estimates; and explore situations where these designs can offer the most substantial savings of time and resources.

\section{Analysis}

\section{Achieving acceptable precision}

A simplified formula used for calculating the width of the one side of the two-sided $95 \%$ confidence interval in cluster surveys is [14]:

$$
\mathrm{d}=\mathrm{Z}^{*} \operatorname{sqrt}(\mathrm{p}(1-\mathrm{p}) * \mathrm{DEFF} / \mathrm{n})
$$

where $d$ = the width of one side of the two-sided $95 \%$ confidence interval

$\mathrm{n}$ = sample size

$\mathrm{p}=$ the prevalence of the outcome being measured

$\mathrm{DEFF}=$ design effect

$\mathrm{Z}=\mathrm{z}$ value (generally 1.96 )

The design effect is the ratio of the variance of the estimate under the actual (for example, cluster) design to the variance of the estimate assuming that the same data have been collected by simple random sampling $[3,10]$. By changing the cluster design, we can modify both the design effect (DEFF) and the sample size (n), while $\mathrm{Z}$ and $p$ will not be affected. The design effect can be presented as a function of the average cluster size (m) and the intracluster correlation coefficient (Rho), which describes the relatedness of cluster data by comparing the variance within clusters with the variance between clusters [21,22]:

$$
\mathrm{DEFF}=1+\operatorname{Rho}(\mathrm{m}-1)
$$

Therefore, the smaller the cluster size, the smaller the design effect. Decreasing the design effect through decreasing the cluster size may, to a certain degree, offset the loss of precision resulting from decreasing the overall sample size [3]. For example, if we observed a design effect of 2 for a certain indicator in a $30 \times 30$ survey, then Rho for this indicator in the surveyed population can be calculated as:

$$
\text { Rho }=(\text { DEFF }-1) /(m-1)=(2-1) /(30-1)=0.0345
$$

Using this value of Rho, we can then calculate an expected DEFF for $33 \times 6,67 \times 3$, and $30 \times 7$ designs:

$$
\begin{aligned}
& \operatorname{DEFF}_{33 \times 6}=1+0.0345^{*}(6-1)=1.1725 \\
& \operatorname{DEFF}_{67 \times 3}=1+0.0345^{*}(3-1)=1.0690 \\
& \operatorname{DEFF}_{30 \times 7}=1+0.0345^{*}(7-1)=1.2070
\end{aligned}
$$


As can be seen, expected design effects for these three designs are substantially lower than the design effect of 2 observed in a $30 \times 30$ survey.

We can now calculate the expected relative change in the width of the confidence interval of the $33 \times 6$ design compared to the $30 \times 30$ design as a ratio of sqrt $\left(\mathrm{DEFF}_{33 \times 6} / \mathrm{n}_{33}\right.$ $\times 6)$ to $\operatorname{sqrt}\left(\mathrm{DEFF}_{30 \times 30} / \mathrm{n}_{30 \times 30}\right)$, since $\mathrm{p}$ and $\mathrm{Z}$ do not change:

$\operatorname{sqrt}(1.1725 / 198) / \operatorname{sqrt}(2 / 900)=0.07695 / 0.04714=1.63$

Therefore, the 95\% confidence interval in the $33 \times 6$ design is expected to be $63 \%$ wider than the $95 \%$ confidence interval in a $30 \times 30$ design.

Table 1 presents similar calculations showing changes in precision for the three designs $(67 \times 3,33 \times 6$, and $30 \times 7)$ compared to a $30 \times 30$ design for design effects of different magnitude. As can be seen, all three designs resulted in much lower design effects than a $30 \times 30$ design. Nevertheless, at lower design effects $(1.25-2.0$ in $30 \times 30$ design), the expected loss in precision (or increase in the width of the confidence interval) was substantial: the width of the confidence intervals increased by $55 \%-95 \%$ compared with the confidence interval of a $30 \times 30$ design. This loss of precision decreased with increasing design effect. Of the three designs, the $67 \times 3$ design consistently resulted in the lowest loss of precision and actually out-performed the $30 \times 30$ design at higher design effects ( 7 and above). The $30 \times 7$ design performed similarly to the $33 \times 6$ design.

Figure 1 presents the half-width of the $95 \%$ confidence interval at different design effect levels in scenarios where the prevalence of the measured outcome is $15 \%$. Again, it can be seen that the losses of precision for all three designs, compared to the $30 \times 30$ design, are the largest at the lowest design effect levels, and they decrease with increasing design effect. Performance of the $30 \times 7$ design is similar to that of the $33 \times 6$, and the $67 \times 3$ design is clearly superior at the highest design effect levels. Although for other prevalence levels of the measured outcome the absolute width of the confidence intervals will differ from those shown in Figure 1, the relative expected differences in precision among different designs will remain the same, as shown previously in Table 1.

In practical terms, these results mean that $67 \times 3,33 \times 6$, and $30 \times 7$ designs are most statistically efficient for measuring outcomes or indicators that have high intracluster correlation, and that they are not as statistically efficient (resulting in substantial loss of precision compared to that of the $30 \times 30$ design) for outcomes with a low degree of clustering. Unfortunately, the body of literature that provides guidance on design effects for different outcomes measured in cluster surveys in emergencies is very limited, and design effects are not routinely included in survey reports. Two recent papers $[3,20]$ provide, however, some insight into commonly seen magnitudes of design effects for indicators frequently measured in emergencies. From these papers, it seems that the prevalence of global acute malnutrition and anemia tend to have the lowest design effects (on the magnitude of 1.2-2.0). Somewhat higher design effects (around 2.5-4.0) are observed for a cumulative 2-week incidence of diarrhea and acute respiratory infection (ARI), and yet higher design effects (5.0 and higher) are observed for vitamin A distribution and vaccination coverage. The highest design effects (10 and higher) are likely to be observed for some household-level

Table I: Expected change in precision for $67 \times 3,33 \times 6$ and $30 \times 7$ designs compared with $30 \times 30$ design

\begin{tabular}{|c|c|c|c|c|c|c|c|}
\hline \multirow{2}{*}{$\begin{array}{l}30 \times 30 \text { design } \\
\text { Observed DEFF }\end{array}$} & \multirow[t]{2}{*}{ Rho } & \multicolumn{2}{|c|}{$67 \times 3$ design } & \multicolumn{2}{|c|}{$33 \times 6$ design } & \multicolumn{2}{|c|}{$30 \times 7$ design } \\
\hline & & $\begin{array}{c}\text { Expected } \\
\text { DEFF }\end{array}$ & $\begin{array}{c}\text { Change in the width } \\
\text { of the } \mathrm{Cl}, 1 \%\end{array}$ & $\begin{array}{c}\text { Expected } \\
\text { DEFF }\end{array}$ & $\begin{array}{c}\text { Change in the width } \\
\text { of the } \mathrm{Cl}, 1 \%\end{array}$ & $\begin{array}{l}\text { Expected } \\
\text { DEFF }\end{array}$ & $\begin{array}{c}\text { Change in the width } \\
\text { of the } \mathrm{Cl}, 1 \%\end{array}$ \\
\hline I & 0 & 1 & +111.6 & I & +113.2 & I & +107.0 \\
\hline 1.25 & 0.0086 & 1.0172 & +90.9 & 1.0431 & +94.7 & 1.0517 & +89.9 \\
\hline 1.5 & 0.01724 & 1.0345 & +75.7 & 1.0862 & +81.4 & 1.1034 & +77.6 \\
\hline 2 & 0.03448 & 1.0690 & +54.7 & 1.1724 & +63.2 & 1.2069 & +60.8 \\
\hline 2.5 & 0.05172 & 1.1034 & +40.6 & 1.2586 & +51.3 & 1.3103 & +49.9 \\
\hline 3 & 0.06897 & 1.1379 & +30.3 & 1.3448 & +42.7 & 1.4138 & +42.1 \\
\hline 4 & 0.10345 & 1.2069 & +16.2 & 1.5172 & +31.3 & 1.6207 & +31.8 \\
\hline 5 & 0.13793 & 1.2759 & +6.9 & 1.6897 & +23.9 & 1.8276 & +25.2 \\
\hline 7 & 0.20690 & 1.4138 & -4.9 & 2.0345 & +14.9 & 2.2414 & +17.1 \\
\hline 10 & 0.31034 & 1.6207 & -14.8 & 2.5517 & +7.7 & 2.8621 & +10.8 \\
\hline
\end{tabular}

\footnotetext{
I Relative expected change in the width of the $95 \%$ confidence interval compared to the $30 \times 30$ design
} 


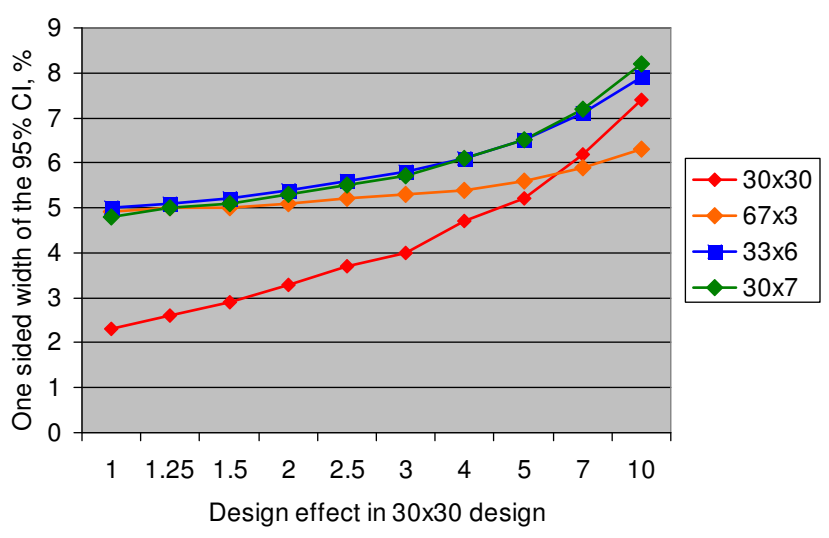

Figure I

The width of the $95 \%$ confidence interval for different cluster designs given $15 \%$ prevalence of the measured outcome.

indicators, such as access to potable water or to latrines [20]. Therefore, $67 \times 3,33 \times 6$, and $30 \times 7$ designs would be most efficient for measuring the most clustered outcomes, such as access to potable water and latrines, vaccination and vitamin A distribution coverage; moderately efficient for measuring cumulative 2 week incidence of ARI or diarrhea; and least efficient for measuring outcomes with the most within-cluster variability, such as global acute malnutrition and anemia.

As a result of small overall sample sizes (around 200 households), $67 \times 3,33 \times 6$, and $30 \times 7$ designs are not likely to provide sufficient levels of precision to measure crude or under-5 mortality rates. For example, assuming that the true under-5 mortality rate in the population is 1.0 per 10,000 per day, a recall period of 3 months, and on average 1 child under 5 years of age per household, the expected number of under- 5 deaths detected in the $33 \times 6$, $30 \times 7$, or $67 \times 3$ surveys is only about 1.8 . This will result not only in a wide confidence interval, even if DEFF for mortality is low, but also in unstable point estimates.

It is also important to consider the minimum desired level of precision in each concrete situation. For example, if in the scenario presented in Figure 1 the investigators are measuring an outcome with an expected design effect of 2 in a $30 \times 30$ design, and consider that achieving the precision of $\pm 6 \%$ is sufficient, they could probably use one of the three designs $(67 \times 3,33 \times 6$, and $30 \times 7)$. If, however, a higher level of precision is required, then the cluster design with the larger overall sample size would need to be considered. In addition, these designs may not provide sufficient precision for the subgroup estimates within the survey sample - for example, when separate estimates are desired for males and females or for different age subgroups within the sample of children under 5 years of age.

\section{Avoiding threats to validity}

One important question is whether designs in which the number of survey subjects per cluster is low ( 3 in $67 \times 3$ to 7 in $30 \times 7$ ) can reliably ensure that the subjects sampled in each cluster are representative of that cluster. With only 3 survey subjects per cluster, such representativeness may be hard to achieve, especially if the EPI method, rather than random sampling, is used for selecting households or subjects within a cluster [14]. It is conceivable that teams that either are pressed for time (if they are expected to complete several clusters in one day), poorly trained, or poorly motivated would be tempted to select 3 or 6 most conveniently or centrally-located houses to achieve the sample size for a given cluster. For some indicators, such "central" bias in sampling may result in biased prevalence estimates. With the $30 \times 30$ design, the teams would often have the whole day to complete one cluster and may be inclined to better adhere to selection procedures prescribed by the EPI method, thus achieving better representativeness. These problems could be overcome to a certain degree if random selection procedures, rather than the EPI method, are used for subject selection. These procedures, however, often require substantial additional time and effort to map or enumerate households within a cluster before random selection can be carried out.

One way to assess the seriousness of this problem is to compare the prevalence estimates obtained from a $67 \times 3$ or a $33 \times 6$ survey to those obtained by using a $30 \times 30$ design, given that the same population is sampled. One recent paper [19] presents such an opportunity. In that paper, $67 \times 3,33 \times 6$, and $30 \times 30$ surveys, all measuring the same outcomes, were conducted in the same population. Prevalence estimates for global acute malnutrition, stunting, underweight, and vaccination coverage were roughly comparable across the three designs. The prevalence of severe acute malnutrition obtained in the $67 \times 3$ survey $(5.7 \%)$ was, however, more that 2.5 times higher than that obtained in $33 \times 6$ and $30 \times 30$ surveys $(2.1 \%$ and $2.3 \%$, respectively). More interestingly, the 2 -week cumulative incidence of diarrhea and fever measured by $33 \times 6$ (31.2\% and 30.7\%, respectively) and $67 \times 3$ (32.5\% and $37.5 \%$, respectively) surveys were substantially higher than estimates for diarrhea and fever obtained from a $30 \times 30$ survey $(24.4 \%$ and $24.8 \%$, respectively). One possible (albeit admittedly speculative) explanation for these differences may be that subjects in $33 \times 6$ and $67 \times 3$ surveys were more likely to be sampled in central locations of the clusters, where higher population density may result in higher incidence of common infections in children than in peripheral locations. Therefore, it would be important to conduct additional, similar studies to assess whether the point estimates for some commonly measured indicators in $33 \times 6$ or $67 \times 3$ surveys consistently and substantially deviate from the estimates 
obtained in $30 \times 30$ surveys and to explore potential reasons if such differences are found. Careful training and supervision of teams conducting these surveys, and use of random rather than EPI procedures for subject or household selection within clusters, will need to be emphasized.

\section{Saving time and resources}

One attractive feature of the survey designs discussed in this paper is the dramatically reduced sample size (from 900 in a $30 \times 30$ survey to around 200). Time and resource savings resulting from this more than four-fold decrease in sample size will likely be one of the main arguments for wider field application of these designs.

In a simplified form, the time needed for a team to complete a survey in the field (assuming that teams do not need to return to their base at the end of each day) can be expressed as:

$$
\mathrm{T}=\mathrm{k}^{*} \mathrm{t}_{1}+(\mathrm{k}+1) * \mathrm{t}_{2}+\mathrm{n}^{*} \mathrm{t}_{3}
$$

where:

$\mathrm{T}$ - total time to complete a survey for one team

$t_{1}$ - average time needed for initial introduction of team to the community in each cluster and initial selection of households within cluster

$\mathrm{t}_{2}$ - average time of travel between clusters or between cluster and base

$t_{3}$ - average time to complete survey for one survey subject/household, including travel between houses

$\mathrm{k}$ - number of clusters

$\mathrm{n}$ - total sample size

Mathematically, it can be shown that relative time savings in $67 \times 3,33 \times 6$, and $30 \times 7$ designs, compared to $30 \times 30$ design, increase with increasing $t_{3}$ and decrease with increasing $t_{1}$ and $t_{2}$. Since the travel time is likely to vary the most from survey to survey, it would be interesting to explore how the time savings change, depending on travel time. Figure 2 presents total time to complete the surveys of different designs for different average travel times $\left(t_{2}\right)$, varying from 15 minutes to 6 hours (assuming both $t_{1}$ and $\mathrm{t}_{3}$ to be 30 minutes). As can be seen, the biggest time savings ( 3 to 4 times less than for the $30 \times 30$ design) are expected when the average travel times between clusters are small (15 minutes to 1 hour). With increasing average travel time between clusters, relative time savings decrease, especially for the $67 \times 3$ design, which at longer

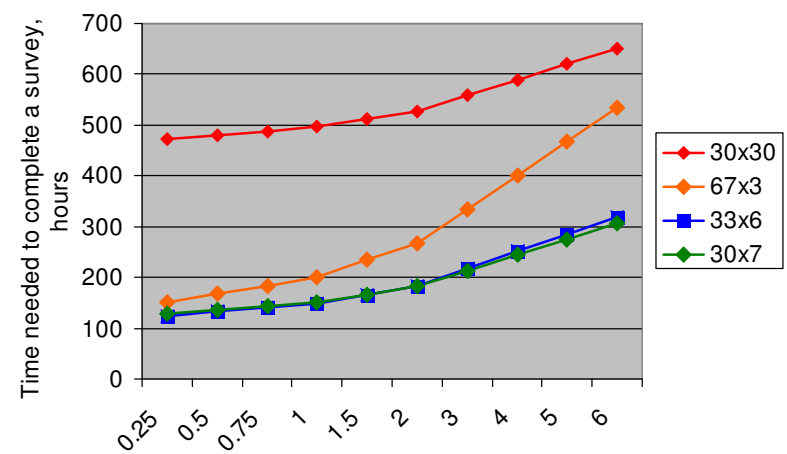

Average travel time between clusters, hours

* Assuming average time to survey one subject is 0.5 hours

\section{Figure 2}

Time needed to complete a survey for different cluster designs depending on average travel time between clusters.

travel times (5-6 hours) offers little time advantage over the $30 \times 30$ design. For longer travel times, the $67 \times 3$ cluster design may present an additional disadvantage of higher fuel costs, since the teams need to travel long distances to 66 instead of 30 different locations. The $33 \times 6$ and $30 \times 7$ designs produce very similar time savings, and at longer travel times (4-6 hours) they would still require less than half the time needed to complete a $30 \times 30$ survey. Therefore, in situations where travel times between clusters are long, logistically $33 \times 6$ and $30 \times 7$ designs would be preferable to the $67 \times 3$ design.

It should be emphasized, however, that the scenario presented above is fairly simple in that it assumes that the team does not need to return to the base at the end of each day and can spend nights in the field, either in or between the clusters it is surveying. Also, it assumes that introduction of the team to the community and initial household selection within a cluster are not very time-consuming. In cases when mapping or enumeration of the households within each cluster needs to be carried out as part of random selection procedures, this initial phase will likely take much longer than 30 minutes. The quality of roads in the survey area and vehicles available to survey teams may have a substantial impact on travel time. Therefore, a time estimation exercise that takes into account specific circumstances of each planned survey needs to be carried out in the planning phase so as to evaluate expected time requirements associated with each of the alternative designs.

\section{Conclusion}

Overall, cluster designs with substantially decreased cluster size and overall sample size, such as the $67 \times 3,33 \times 6$, 
and $30 \times 7$ designs discussed in this paper, may offer substantial time and cost savings compared to the traditional $30 \times 30$ design, and they may provide levels of precision that are acceptable (or at least comparable to the $30 \times 30$ design) for measuring some common outcomes of interest in humanitarian emergencies. This is particularly the case for outcomes that tend to have high intracluster homogeneity, such as vaccination coverage, vitamin A supplementation coverage, or access to safe water. Using these designs to measure outcomes that are normally less clustered (e.g, global acute malnutrition) may result in a sizeable loss in precision (resulting in confidence intervals 1.5 to 2 times wider than those obtained with the $30 \times 30$ design), and may therefore need to be carefully considered vis-à-vis context-specific minimum requirements for precision. Because of the small overall sample size, these designs are not likely to provide sufficient precision to measure crude or under-5 mortality rates. The question of the ability of these designs to consistently provide accurate (or unbiased) point estimates for key indicators routinely measured in humanitarian emergencies remains open, and it is subject to further investigation. Good training and supervision of the teams implementing these designs in the field and use of random rather than EPI methods for household selection will be important for ensuring selection of representative samples within clusters.

Because of substantially reduced overall sample size, these designs will likely result in substantial (2-4 times compared to $30 \times 30$ design) time savings. The magnitude of these time savings will depend on several factors, including the average time needed to complete a survey for one survey subject or household, the average time to select households within a cluster, and the average time needed to travel between clusters. The $67 \times 3$ design will provide the least time savings relative to the $30 \times 30$ design, and it may be logistically difficult to implement if travel distances between clusters are large. The $33 \times 6$ and $30 \times 7$ designs are expected to perform similarly to each other, both in terms of statistical efficiency and in terms of time required to complete the survey.

Finally, despite several proposed "standard" designs that can provide the minimum required level of accuracy and precision, some of which were discussed in this paper, the best solution is to calculate sample sizes individually for each planned survey, taking into account the specific indicators being measured, context-specific levels of precision required, logistic and security concerns, availability of suitable personnel, and other factors. Many surveys in emergencies strive to measure multiple outcomes, including nutritional status, mortality, water and sanitation indicators, access to feeding programs and food aid, among others. In these situations, sample sizes to achieve acceptable levels of precision need to be calculated for each of the key indicators [14], and the best solution in terms of sampling design and sample size needs to be determined based on this information. It is important to continue building technical capacity in cluster survey design within organizations conducting cluster surveys in humanitarian emergencies [15-17]. Several important initiatives (Standardized Monitoring and Assessment of Relief and Transitions (SMART), Health and Nutrition Tracking Service) and recently published survey manuals $[13,14]$ should prove instrumental in achieving this goal.

\section{Competing interests}

The author declares that he has no competing interests.

\section{Authors' contributions}

OOB designed the study, performed analysis, wrote and revised the manuscript.

\section{Acknowledgements}

I thank Curtis Blanton, M.S., for his valuable comments to the earlier version of this manuscript.

\section{Disclaimer:}

The findings and conclusions in this report are those of the author and do not necessarily represent the views of the Centers for Disease Control and Prevention.

\section{References}

I. Brogan D, Flagg EW, Deming M, Waldman R: Increasing the accuracy of the expanded programme on immunization's cluster survey design. Ann Epidemiol 1994, 4:302-31I.

2. Bostoen K, Bilukha OO, Fenn B, Morgan O, Tam C, ter Veen A, Checchi $F$ : Methods for health surveys in difficult settings: charting progress, moving forward. Emerg Themes Epidemiol 2007, 4:13.

3. Kaiser R, Woodruff BA, Bilukha O, Spiegel PB, Salama P: Using design effects from previous cluster surveys to guide sample size calculation in emergency settings. Disasters 2006, 30:|99-2।I.

4. Degomme O, Guha-Sapir D: Mortality and nutrition surveys by Non-Governmental organizations. Perspectives from the CE-DAT database. Emerg Themes Epidemiol 2007, 4: II.

5. Lemeshow S, Tserkovnyi AG, Tulloch JL, Dowd JE, Lwanga SL, Keja J: A computer simulation of the EPI survey strategy. Int J Epidemiol 1985, I4:473-48I.

6. Henderson RH, Sundaresan T: Cluster sampling to assess immunization coverage: a review of experience with a simplified sampling method. Bull WHO 1982, 60:253-260.

7. Assefa F, Jabarkhil MZ, Salama P, Spiegel P: Malnutrition and mortality in Kohistan district, Afghanistan, April 200 I. JAMA 200I, 2 I :2723-2728.

8. Spiegel PB, Salama P: War and mortality in Kosovo, 1998-99: an epidemiological testimony. Lancet 2000, 355:2204-2209.

9. Woodruff BA, Blanck HM, Slutsker L, Cookson ST, Larson MK, Duffield A, Bhatia R: Anaemia, iron status and vitamin A deficiency among adolescent refugees in Kenya and Nepal. Public Health Nutrition 2006, 9:26-34.

10. Katz J, Zeger SL: Estimation of design effects in cluster surveys. Ann Epidemiol 1994, 4:295-301.

II. United Nations Administrative Committee on Coordination - SubCommittee on Nutrition: Report of a workshop on the improvement of the nutrition of refugees and displaced people in Africa Machakos, Kenya; 1995. 
12. de Ville de Goyet C, Seaman J, Geijer U: The Management of Nutritional Emergencies in Large Populations Geneva: World Health Organization; 1978.

13. Standardized monitoring and assessment of relief and transitions (SMART). Measuring mortality, nutritional status and food security in crisis situations. Version I [http://www.smart indicators.org]

14. Centers for Disease Control and Prevention (CDC) and World Food Programme: A Manual: Measuring and Interpreting Malnutrition and Mortality Rome: World Food Programme; 2005.

15. Prudhon C, Spiegel P: A review of methodology and analysis of nutrition and mortality surveys conducted in humanitarian emergencies from October 1993 to April 2004. Emerg Themes Epidemiol 2007, 4: 10.

16. Spiegel P: Who should be undertaking population-based surveys in humanitarian emergencies? Emerg Themes Epidemiol 2007, 4: 12 .

17. Spiegel P, Salama P, Maloney S, Veen A van der: Quality of malnutrition assessment surveys conducted during famine in Ethiopia. JAMA 2004, 292:613-618.

18. Boss LP, Toole MJ, Yip R: Assessments of mortality, morbidity, and nutritional status in Somalia during the $1991-1992$ famine. Recommendations for standardization of methods. JAMA 1994, 272:37I-6.

19. Deitchler M, Valdez JJ, Egge K, Fernandez S, Hennigan M: A field test of three LQAS designs to assess the prevalence of acute malnutrition. Int J Epidemiol 2007, 36:858-864.

20. Deitchler M, Deconinck H, Bergeron G: Precision, time and cost: a comparison of three sampling designs in an emergency setting. Emerg Themes Epidemiol 2008, 5:6.

21. Kish L: Survey Sampling New York: John Wiley and Sons, Inc; 1965

22. Killip S, Mahfoud Z, Pearce K: What is an intracluster correlation coefficient? Crucial concepts for primary care researchers. Ann Fam Med 2004, 2:204-208.

Publish with Biomed Central and every scientist can read your work free of charge

"BioMed Central will be the most significant development for disseminating the results of biomedical research in our lifetime. "

Sir Paul Nurse, Cancer Research UK

Your research papers will be:

- available free of charge to the entire biomedical community

- peer reviewed and published immediately upon acceptance

- cited in PubMed and archived on PubMed Central

- yours - you keep the copyright

Submit your manuscript here:

http://www.biomedcentral.com/info/publishing_adv.asp 